\title{
RELAÇÃO ENTRE A ALTURA DE INUNDAÇÃO, RIQUEZA ESPECÍFICA DE PLANTAS E O TAMANHO DE CLAREIRAS NATURAIS EM UMA FLORESTA INUNDÁ VEL DE IGAPÓ, NA AMAZÔNIA CENTRAL ${ }^{1}$
}

\author{
Leandro Valle Ferreira ${ }^{2}$ e Samuel S. Almeida ${ }^{2}$
}

\begin{abstract}
RESUMO - Apesar do considerável número de estudos publicados sobre clareiras em regiões tropicais, nenhum deles foi publicado sobre a regeneração de espécies de plantas em clareiras naturais nas florestas inundadas da Amazônia. Essas florestas apresentam forte flutuação do nível dos rios, que pode variar em até 15 m, entre as estações de seca e cheia, inundando extensas áreas ao longo de rios e igarapés. O objetivo deste estudo foi determinar se diferenças na posição das clareiras no gradiente de inundação e conseqüentemente no tempo de inundação anual poderiam afetar os padrões de tamanho, riqueza e composição específica em clareiras naturais. Também, foram amostradas 10 clareiras naturais situadas em diferentes posições do gradiente de inundação do rio Tarumã-Mirim, um afluente do rio Negro, no Estado do Amazonas, Brasil. Houve aumento significativo na área das clareiras, variando de 101 a $1.001 \mathrm{~m}^{2}$, em relação ao aumento do gradiente de inundação. Houve, ainda, incremento significativo no número total de espécies regenerando nas clareiras, variando de 14 a 51 espécies, em relação ao aumento do gradiente de inundação. A composição de espécies regenerando nas clareiras foi bastante relacionada com sua posição no gradiente de inundação, e clareiras situadas em regiões sujeitas a longos períodos de inundação são colonizadas principalmente por espécies com grande tolerância à inundação, enquanto as clareiras situadas em regiões submetidas a períodos curtos de inundação são, sobretudo, colonizadas por espécies pouco tolerantes à inundação. Concluindo, a área total, o número e a composição das espécies nas clareiras da floresta de igapó amostrada neste estudo foram relacionados com o gradiente de inundação, demonstrando que o tempo anual de inundação influiu nos parâmetros analisados.
\end{abstract}

Palavras-chave: Amazônia, clareiras, floresta de igapó e regeneração.

\section{RELATIONSHIP BETWEEN FLOODING LEVEL, PLANT SPECIES DIVERSITY AND TREE FALL GAP SIZE IN A SEASONALLY FLOODED FOREST IN CENTRAL AMAZONIA, BRAZIL}

\begin{abstract}
Despite the considerable number of studies published about tree gaps in tropical regions, none of them addresses the regeneration of plant species in natural gaps in the Amazonian seasonally flooded forests. These forests undergo a large fluctuation of the water level which may vary up to 15 meters between the high and low water seasons, flooding extensive areas along rivers and streams. The objective of this study was to determine whether differences in the duration of annual flooding could affect the parameters of size, species diversity, and composition in natural gaps. This study sampled 10 natural gaps in black water floodplain forests of different areas and flood levels along the Tarumã-Mirim River, a tributary of the Rio Negro, in
\end{abstract}

\footnotetext{
${ }^{1}$ Recebido em 13.02.2004 e aceito para publicação em 20.04.2005.

${ }^{2}$ Museu Paraense Emílio Goeldi -MPEG/CCTE/CBO. Avenida Perimetral 1901 - Bairro Terra Firme -66077-530. BelémPA, Brasil. Email: <lvferreira@ museu-goeldi.br>,<samuel@museu-goeldi.br>.
} 
the State of Amazonas, Brazil. There was a significant increase in gap size, varying from 101 to $1.001 \mathrm{~m} 2$, in relation to the level of flooding. There was also a significant increase in species diversity, varying from 14 to 51 species, depending upon the increase in the level of flooding. The species composition in the gaps was closely related to the level of flooding, resulting in gaps located in areas subjected to a long flooding period being colonized mainly by species with a high flooding tolerance, whereas gaps located in regions of short periods of inundation were mainly colonized by species with a low flooding tolerance. In conclusion, the gap size, diversity, and floristic composition within the gaps of the black water flooded forests sampled in this study were related to the level offlooding, demonstrating that that annual flooding duration influenced these parameters.

Key Words: Amazon, gaps, igapó forests and plant regeneration.

\section{INTRODUÇÃO}

O regime de perturbações em micro e pequena escala são um dos fatores mais importantes para se entender a dinâmica da regeneração natural de espécies nos trópicos (UHL et al., 1988; WHITMORE, 1985). Na região tropical, a maioria das perturbações naturais é causada pela queda de árvores e de copas provocadas pela ação de chuvas e ventos fortes, abrindo o dossel da floresta e formando as clareiras (WHITMORE, 1978; UHL et al., 1978; BROKAW, 1985b; ALMEIDA, 1989). Apesar do considerável número de estudos publicados sobre clareiras em regiões tropicais (HARTSHORN, 1978; UHL et al., 1978; WHITMORE, 1978, 1985; BROKAW, 1982, 1985ab), poucos foram realizados em florestas amazônicas, como o de Almeida (1989), que pesquisou os fatores ligados à formação e regeneração de espécies em clareiras naturais em uma floresta de terra firme próximo a Manaus (Amazonas, Brasil). Contudo, não existem estudos publicados sobre os fatores bióticos ou abióticos ligados à formação e regeneração de espécies de plantas em clareiras naturais nas florestas inundadas da Amazônia brasileira.

As florestas inundadas na Amazônia apresentam forte sazonalidade devido à flutuação cíclica no nível das águas dos rios, que podem atingir $14 \mathrm{~m}$, resultando em inundações de vastas áreas ao longo de suas margens (JUNK, 1989). Essas florestas têm sido descritas em termos de características estruturais, fisionômicas e florísticas (DUCKE et al., 1953; RODRIGUES, 1961; TAKEUCHI,1962; KEEL et al., 1979; PIRES et al., 1985; AYRES, 1986; PRANCE, 1987; JUNK, 1989; FERREIRA, 1997, 2000). Prance (1979) definiu sete tipos principais de formações vegetais inundadas na Amazônia. Desses tipos, os mais importantes são as florestas periodicamente inundadas por rios de água branca, definidas como florestas de várzeas, e aquelas periodicamente inundadas por rios de "preta" ou "clara", denominadas florestas de igapós.

Os tipos de vegetação de várzeas (florestas, campos ou restingas) ocorrem em formações do Período Quaternário Recente, apresentando sedimentos ricos em nutrientes e com elevada dinâmica hidrogeomorfológica. Por seu turno, as florestas de igapós e os tipos de vegetação não florestal ocorrem em formações mais antigas do Período Terciário ou Pré-Cambriano com baixos níveis de nutrientes (FITTKAU, 1971; AYRES, 1986).

Nas florestas inundadas, as variações topográficas podem resultar em diferenças na amplitude e tempo de inundação anual, fatores determinantes nos padrões observados de riqueza, diversidade e composição das espécies (AYRES, 1986; JUNK, 1989; FERREIRA, 1997, 2000). Poucos estudos relacionam o impacto do tempo de inundação anual na dinâmica da regeneração e mortalidade de espécies vegetais nas florestas inundadas da Amazônia. Nas florestas de várzea, Salo et al. (1986) relataram que condições hipóxicas e de erosão na Amazônia peruana podem provocar profundas alterações na estrutura do solo, provocando a mortalidade de árvores. Ayres (1993) relatou que 78\% da mortalidade de árvores em uma floresta de várzea no rio Japurá, Brasil, foi provocada pela ação erosiva da água no sistema radicular das árvores. Campbell et al. (1992) registraram taxas de mortalidade anuais variando de 1,5 a $2 \%$ em parcelas de 1 ha em uma floresta de várzea no rio Juruá (Amazonas, Brasil). Nas florestas de igapó, verificou -se a mortalidade de árvores nos rios Negro e Jaú (Amazonas, Brasil), onde regiões são submetidas a longos períodos de inundação, resultando em extensas faixas de árvores mortas em pé, decorrente da falta 
de oxigenação das raízes em anos com cheias prolongadas (PIEDADE, 1985; JUNK, 1989).

O objetivo do presente trabalho foi determinar se diferenças no período de inundação influenciada pela topografia do terreno também poderiam afetar os padrões de estrutura de tamanho, riqueza e composição de espécies em clareiras naturais em uma floresta de igapó do Igarapé Tarumã-Mirim, Amazonas, Brasil.

\section{MATERIAL E MÉTODOS}

\section{1. Área de estudo}

Está localizada no baixo curso do igarapé TarumãMirim, um afluente da margem esquerda do rio Negro $\left(3^{\circ} 02^{\prime} \mathrm{S} ; 60^{\circ} 10^{\prime} \mathrm{W}\right.$ ) a cerca de $20 \mathrm{~km}$ a nordeste de Manaus, Amazonas (Figura 1).

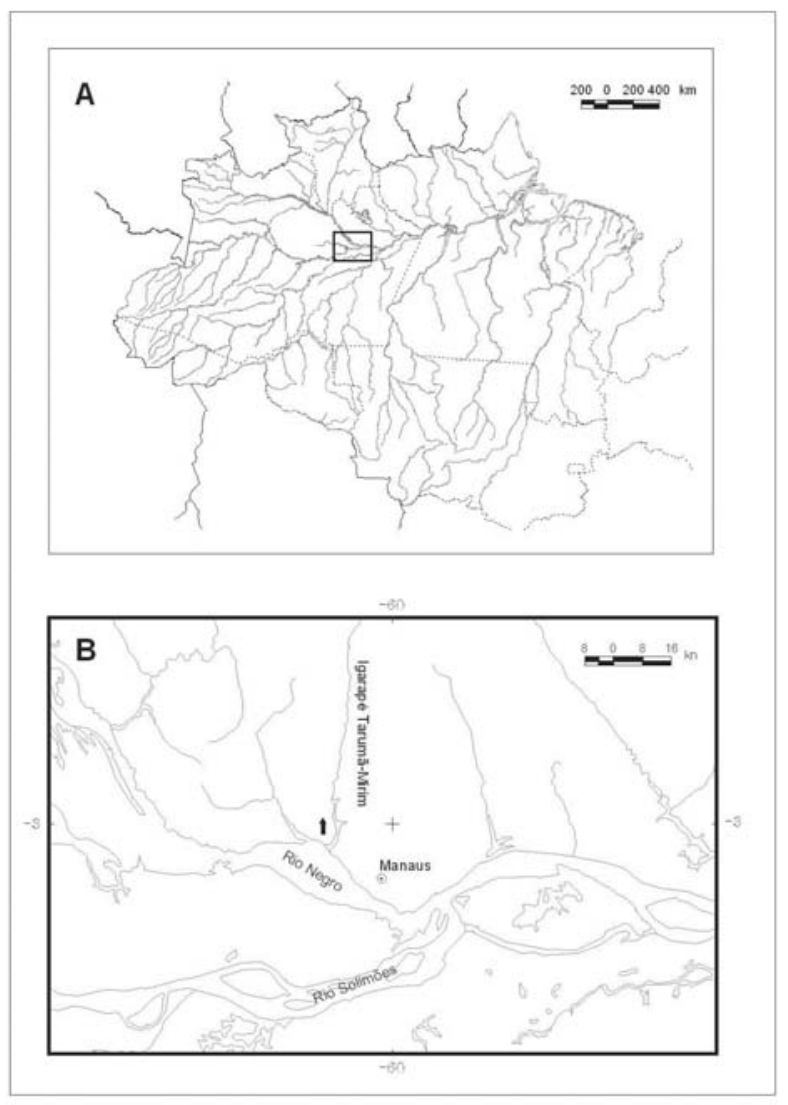

Figura 1 - Mapa da Amazônia Legal brasileira (A) e detalhe da área de estudo (seta) no igarapé Tarumã-Mirim próximo de Manaus, Amazonas, Brasil (B).

Figure 1 -Map of the Brazilian Legal Amazonia (A) and detail of the study site (arrow) in the Tarumã-Mirim stream near Manaus, Amazonas, Brazil (B).
O período de inundação do rio Negro normalmente ocorre no final de novembro a princípio de junho, enquanto o período de vazante ocorre entre o fim de junho e o começo de novembro.

A área de estudo apresentava um gradiente topográfico variando de locais próximo à margem do rio até a transição com a floresta de terra firme, resultando em níveis de enchente que variam de 1 a $6 \mathrm{~m}$ de altura e períodos de inundação anual oscilando de dois a nove meses (ADIS, 1984; JUNK, 1989).

\subsection{Coleta de dados}

Para demarcação dos limites das 10 clareiras amostradas neste estudo, utilizou a definição de Brokaw (1985a), em que clareiras são "aberturas naturais do dossel florestal formada pela queda de árvores, delimitadas pelas extremidades das copas das árvores laterais". Esse limite foi formado pela área compreendida pela projeção das copas das árvores laterais, correspondendo exatamente à área com dossel aberto.

Para determinar a área total das clareiras, foram colocados um piquete central no centro da clareira e oito piquetes laterais no limite da clareira com ângulo de $45^{\circ}$ entre si, formando, dessa forma, oito triângulos. A área total da clareira foi calculada a partir da soma das áreas dos 8 triângulos (Figura 2).

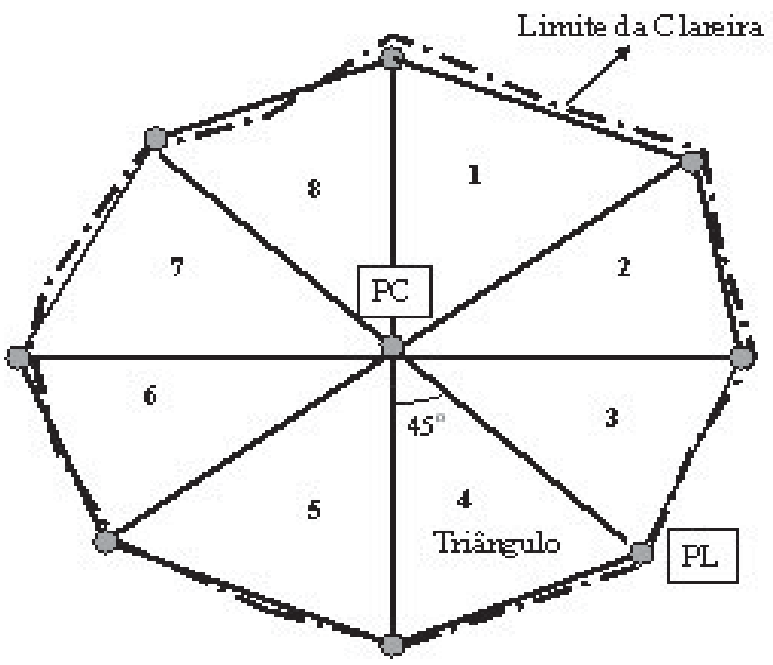

Figura 2 - Diagrama mostrando o método utilizado neste estudo para calcular a área total da clareira usando a soma da área dos triângulos ( $\mathrm{PC}=$ Piquete central e PL $=$ Piquete lateral).

Figure 2-Diagram showing the method used in this study to calculate the total size gap $(P C=$ Central pole and $P L=$ lateral pole) using the sum of the triangle area.

R. Árvore, Viçosa-MG, v.29, n.3, p.445-453, 2005 
Dentro de cada clareira foi realizado um levantamento qualitativo das espécies presentes com menos de 1 $\mathrm{m}$ de altura. Esse levantamento foi dividido em duas partes: 1) contagem de todas as espécies presentes na clareira e 2) amostragem qualitativa de todas as espécies, em uma parcela de $10 \times 10 \mathrm{~m}$, no centro da clareira.

\subsection{Análise de dados}

Utilizou-se a análise de regressão simples para testar a hipótese de existência ou não de diferenças quanto ao tamanho da clareira, ao número de total de espécies e ao número de espécies nas parcelas de 100 $\mathrm{m}^{2}$ (variáveis dependentes), em relação ao gradiente de inundação da área de estudo (variável independente).

\section{RESULTADOS E DISCUSSÃO}

Houve diferença significativa no aumento da área das clareiras em relação ao incremento do gradiente de inundação da área de estudo $\left(\mathrm{F}_{[1,8]}=11,42 ; \mathrm{p}=0,01\right.$; e $\left.r^{2}=0,53\right)$ (Figura 3).

Houve diferença significativa entre o número total de espécies e o número total de espécies regenerando na parcela amostrada no centro da clareira, em relação ao gradiente de inundação da área de estudo $\left(\mathrm{F}_{[1,8]}=49,43\right.$; $\left.\mathrm{p}=0,0001 ; \mathrm{r}^{2}=0,86\right)$ e $\left(\mathrm{F}_{[1,8]}=30,61 ; \mathrm{p}=0,001 ; \mathrm{r}^{2}=0,79\right)$, respectivamente (Figuras 4 e 5 ).

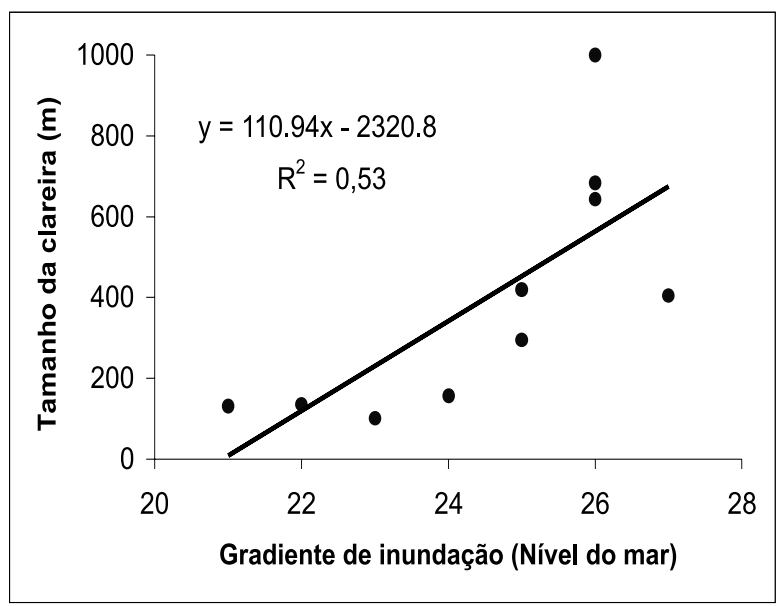

Figura 3 - Relação do tamanho das clareiras amostradas neste estudo em relação ao gradiente de inundação.

Figure 3 - Relationship between gap sizes sampled in the study and the flooding gradient.

R. Árvore, Viçosa-MG, v.29, n.3, p.445-453, 2005

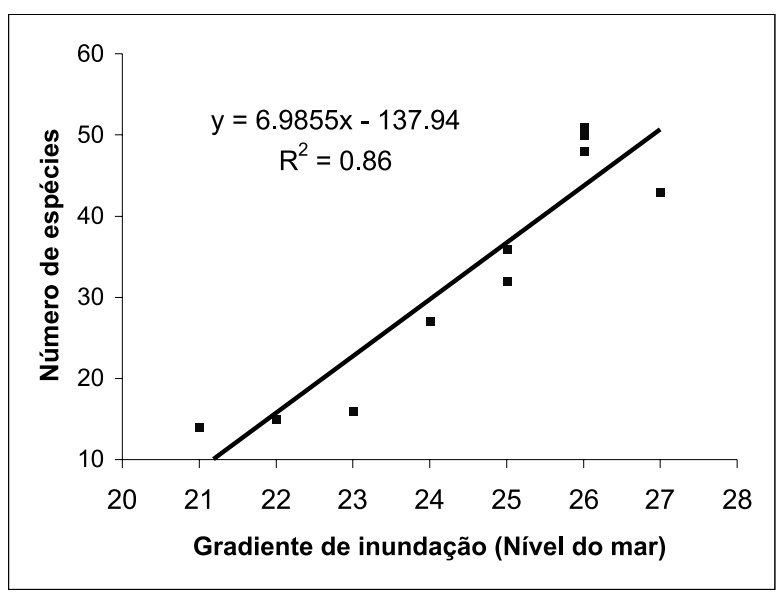

Figura 4 - Relação do tamanho das clareiras amostradas neste estudo em relação ao gradiente de inundação.

Figure 4-Relationship between gap sizes sampled in the study and the flooding gradient.

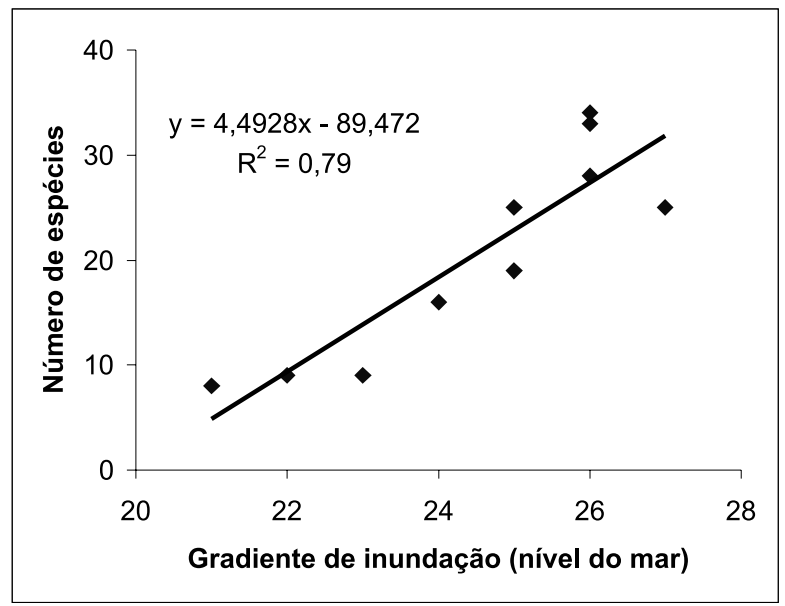

Figura 5 - Relação do tamanho das clareiras amostradas neste estudo em relação ao gradiente de inundação.

Figure 5 - Relationship between gap sizes sampled in the study and the flooding gradient.

A variação no tamanho e idade das clareiras tem grande importância na riqueza e florística sucessional (WHITMORE, 1978; ALMEIDA, 1989). Essa importância é decorrente da variação no nível da luz, temperatura e umidade em relação à área total da clareira (WHITMORE, 1978; BROKAW, 1982; ALMEIDA, 1989; CARVALHO e OLIVEIRA-FILHO 2001).

A área média registrada das clareiras amostradas neste estudo $\left(397,4 \mathrm{~m}^{2}\right)$ é considera elevada quando 
comparada com a de outros estudos realizados na região tropical não-inundável, como a floresta de terra firme da Amazônia e as florestas montanhosas e semideciduais da mata Atlântica (Tabela 1).

Clareiras com áreas superiores às encontradas neste estudo são citadas somente em regiões onde a ocorrência de perturbações naturais catastróficas, como ciclones, furacões ou atividades vulcânicas, é comum (WHITMORE, 1985). Dessa forma, além dos fatores primários de perturbações naturais na formação das clareiras em ambientes não submetidos a inundações, como vento, chuva e relevo (UHL, 1978), na floresta de igapó da área de estudo, o ciclo anual de inundação pode ser um dos fatores adicionais de perturbação, explicando, dessa forma, a maior área média das clareiras desta pesquisa (Tabela 1 ).

O aumento da riqueza de espécies em clareiras em relação à sua área total é um padrão comum na região tropical (HARTSHORN, 1978; WHITMORE, 1978; BROKAW, 1980; UHL et al., 1988; ALMEIDA, 1989; OLIVEIRA-FILHO 2001).

A diferença significativa no número de espécies em relação ao gradiente de inundação é provavelmente resultante das diferenças nos períodos de inundação ao longo do ano da área de estudo (FERREIRA, 1991). Os padrões de riqueza, diversidade e composição de espécies nas florestas inundadas na Amazônia brasileira parecem ser dependentes da duração da inundação (JUNK, 1989; FERREIRA, 1991; AYRES, 1993). Campbell et al. (1992), Junk (1989) e Ayres (1993) verificaram aumento no número de espécies com a diminuição da inundação nas florestas inundadas de várzea, enquanto Keel et al. (1979) e Ferreira (1997, 2000) verificaram o mesmo padrão nas florestas de igapó.

A área e o número total de espécies amostradas nas clareiras deste estudo foram correlacionados com o gradiente de inundação. Dessa forma, o aumento da riqueza poderia ser associado ao incremento do tamanho da clareira e não ao aumento do gradiente de inundação. Contudo, a padronização do esforço de amostragem, usando uma parcela de $100 \mathrm{~m}^{2}$ no centro de cada clareira, evidenciou o considerável efeito do aumento do gradiente de inundação com o crescimento da riqueza de espécies regenerando nas clareiras.

A composição florística é bastante distinta entre as clareiras situadas em diferentes pontos do gradiente de inundação da área de estudo. As clareiras submetidas a longos períodos de inundação são colonizadas principalmente por espécies restritas às cotas topográficas mais baixas da floresta de igapó, como Symmeria paniculata, Couepia paraensis, Licania apetala, Crudia amazonica e Eschweilera tenuifolia (Tabela 2).

Tabela 1 - Área média das clareiras amostradas em estudos realizados em regiões tropicais Table 1 -Mean area of tree fall gaps in several studies carried out in tropical regions

\begin{tabular}{lcl}
\hline Local do Estudo & Área Média $\left(\mathrm{m}^{2}\right)$ & Fonte \\
\hline Costa do Marfim (África) & 171,2 & Bonnis (1980) \\
MPassa (Gabão) & 248,0 & Florence $(1981)$ \\
La Selva (Costa Rica) & 125,0 & Hartshorn $(1978)$ \\
La Selva (Costa Rica) & 161,0 & Sanford (1986) \\
Barro Colorado (Panamá) & 84,8 & Brokaw (1982) \\
Los Tuxtlas (México) & 200,0 & Torquebiau (1981) \\
Amazonas (Brasil) & & Almeida (1989) \\
$\quad$ Floresta de terra firme & 179,1 & \\
Amazonas (Brasil) & & \\
$\quad$ Floresta de igapó & 397,4 & \\
$\quad$ Este estudo & & Almeida ${ }^{1}$ \\
Pará (Brasil) $\quad 115,0$ & \\
$\quad$ Floresta de igapó & 145,0 & Tabarelli e Mantovani (1999) \\
Floresta de várzea & & Martins e Rodrigues (1999) \\
$\quad$ Fão Paulo (Brasil) & $30,3-500,5$ &
\end{tabular}

${ }^{1}$ Dados não publicados. 
Tabela 2 - Listagem e freqüência das espécies de plantas regenerando na parcela do centro das clareiras, na floresta de igapó do igarapé Tarumã-Mirim, Amazonas, Brasil

Table 2 - Species checklist and frequency of plants regrowing in the central plot of the tree fall gaps of the black water floodplain forest at Tarumã-Mirim stream, Amazonas, Brazil

\begin{tabular}{|c|c|c|c|c|c|c|c|c|c|c|c|c|}
\hline Espécie & Família & Freq & 1 & 2 & 3 & 4 & 5 & 6 & 7 & 8 & 9 & 10 \\
\hline Acmanthera latifolia (Adr. Jus.) Griseb. & Malpighiaceae & 7 & & $\mathrm{x}$ & $\mathrm{x}$ & $\mathrm{x}$ & & $\mathrm{x}$ & $\mathrm{x}$ & $\mathrm{x}$ & $\mathrm{x}$ & \\
\hline Acosmium nitens (Vogel) Yakovlev & Fabaceae & 1 & & & & & & & $\mathrm{x}$ & & & \\
\hline Alchornea schomburgkii Klotzsch & Euphorbiaceae & 2 & & & & & & $\mathrm{x}$ & $\mathrm{x}$ & & & \\
\hline Aldina latifolia Spruce ex Benth & Fabaceae & 8 & $\mathrm{x}$ & & $\mathrm{x}$ & & $\mathrm{x}$ & $\mathrm{x}$ & $\mathrm{x}$ & $\mathrm{x}$ & $\mathrm{x}$ & $\mathrm{x}$ \\
\hline Anacampta rupicola (Benth.) Markgr. & Apocynaceae & 2 & & & & & & & & & $\mathrm{x}$ & $\mathrm{x}$ \\
\hline Annonaceae Indet. & Annonaceae & 1 & & & & & & & $\mathrm{x}$ & & & \\
\hline Apocynaceae Indet. & Apocynaceae & 1 & & & & & & & $\mathrm{x}$ & & & \\
\hline Arrabideaea sp. 1 & Bignoniaceae & 4 & & & & $\mathrm{x}$ & $\mathrm{x}$ & $\mathrm{x}$ & $\mathrm{x}$ & & & \\
\hline Aspidosperma carapanauba Pichon & Apocynaceae & 2 & & & & & $\mathrm{x}$ & & & & & \\
\hline Astrocaryum jauari Mart. & Arecaceae & 10 & $\mathrm{x}$ & $\mathrm{x}$ & $\mathrm{x}$ & $\mathrm{x}$ & $\mathrm{x}$ & $\mathrm{x}$ & $\mathrm{x}$ & $\mathrm{x}$ & $\mathrm{x}$ & $\mathrm{x}$ \\
\hline Bactris maraja Mart. & Arecaceae & 2 & $\mathrm{x}$ & & $\mathrm{x}$ & & & & & & & \\
\hline Bambusa sp. 1 & Poaceae & 4 & & & & & & $\mathrm{x}$ & $\mathrm{x}$ & & $\mathrm{x}$ & $\mathrm{x}$ \\
\hline Bignoniaceae Indet. & Bignoniaceae & 1 & & & & & & & $\mathrm{x}$ & & & \\
\hline Calophyllum brasiliense Cambess. & Clusiaceae & 1 & & & & & & & $\mathrm{x}$ & & & \\
\hline Campsiandra comosa Benth. & Fabaceae & 1 & & & & & & & $\mathrm{x}$ & & & \\
\hline Caraipa grandifolia Mart. & Clusiaceae & 6 & & & & $\mathrm{x}$ & & $\mathrm{x}$ & $\mathrm{x}$ & $\mathrm{x}$ & $\mathrm{x}$ & $\mathrm{x}$ \\
\hline Caryocar microcarpum Ducke & Caryocaraceae & 4 & & & & & & $\mathrm{x}$ & & $\mathrm{x}$ & $\mathrm{x}$ & $\mathrm{x}$ \\
\hline Casearia sp. 1 & Flacourtiaceae & 5 & & & & $\mathrm{x}$ & $\mathrm{x}$ & $\mathrm{x}$ & $\mathrm{x}$ & $\mathrm{x}$ & & \\
\hline Cecropia sp. 1 & Cecropiaceae & 1 & & & & & & & $\mathrm{x}$ & & & \\
\hline Clathrotropis nitida (Benth.) Harms. & Fabaceae & 4 & & $\mathrm{x}$ & & & & & & $\mathrm{x}$ & $\mathrm{x}$ & $\mathrm{x}$ \\
\hline Combretum sp. 1 & Combretaceae & 3 & & & & & & & $\mathrm{x}$ & $\mathrm{x}$ & & $\mathrm{x}$ \\
\hline Compositae Indet. & Compositae & 1 & & & & & & & $\mathrm{x}$ & & & \\
\hline Connarus sp. 1 & Connaraceae & 3 & & $\mathrm{x}$ & & & & $\mathrm{x}$ & & $\mathrm{x}$ & & \\
\hline Couepia paraensis Benth. ex Hook. f. & Chrysobalanaceae & 2 & $\mathrm{x}$ & & $\mathrm{x}$ & & & & & & & \\
\hline Crudia amazonica Spruce ex Benth & Caesalpiniaceae & 5 & $\mathrm{x}$ & $\mathrm{x}$ & $\mathrm{x}$ & & & & $\mathrm{x}$ & $\mathrm{x}$ & & \\
\hline Cybianthus sp. 1 & Myrsinaceae & 1 & & & & & & & & & $\mathrm{x}$ & \\
\hline Cynometra spruceana Benth. & Fabaceae & 5 & & & & & $\mathrm{x}$ & $\mathrm{x}$ & $\mathrm{x}$ & $\mathrm{x}$ & & $\mathrm{x}$ \\
\hline Cyperus sp. 1 & Cyperaceae & 7 & & & $\mathrm{x}$ & $\mathrm{x}$ & $\mathrm{x}$ & $\mathrm{x}$ & & $\mathrm{x}$ & $\mathrm{x}$ & $\mathrm{x}$ \\
\hline Dalbergia inundata Spruce ex Benth & Fabaceae & 1 & $\mathrm{x}$ & & & & & & & & & \\
\hline Dalbergia sp. 1 & Fabaceae & 2 & & & & & & $\mathrm{x}$ & & & $\mathrm{x}$ & \\
\hline Dalbergia sp. 2 & Fabaceae & 1 & & & & & & & & & & $\mathrm{x}$ \\
\hline Desmoncus sp. 1 & Arecaceae & 1 & & & & & & & & & & $\mathrm{x}$ \\
\hline Diospyrus sp. 1 & Ebenaceae & 4 & & & & & $\mathrm{x}$ & & & $\mathrm{x}$ & $\mathrm{x}$ & $\mathrm{x}$ \\
\hline Discocarpus Klotzsch & Euphorbiaceae & 2 & & & & & & & & $\mathrm{x}$ & $\mathrm{x}$ & \\
\hline Ebenaceae Indet. & Ebenaceae & 2 & & & & & & & & $\mathrm{x}$ & $\mathrm{x}$ & \\
\hline Eschweilera tenuifolia (O. Berg) Miers. & Lecythidaceae & 2 & $\mathrm{x}$ & $\mathrm{x}$ & & & & & & & & \\
\hline Garcinia sp. 1 & Clusiaceae & 1 & & & & & & & & $\mathrm{x}$ & & \\
\hline Licania apetala (E. Mey.) Fritsch & Chrysobalanaceae & 3 & $\mathrm{x}$ & $\mathrm{x}$ & $\mathrm{x}$ & & & & & & & \\
\hline Nectandra sp. 2 & Lauraceae & 5 & & & & $x$ & & $x$ & $x$ & $x$ & $x$ & \\
\hline Pouteria elegans (A. DC.) Baehni & Sapotaceae & 7 & & $\mathrm{x}$ & $\mathrm{x}$ & & $\mathrm{x}$ & $\mathrm{x}$ & $\mathrm{x}$ & $\mathrm{x}$ & $\mathrm{x}$ & \\
\hline Ocotea sp. 1 & Lauraceae & 2 & & & & & & & & $\mathrm{x}$ & $\mathrm{x}$ & \\
\hline Pariana sp. 1 & Poaceae & 2 & & & & & & & & & $\mathrm{x}$ & $\mathrm{x}$ \\
\hline Parkia auriculata Spruce ex Benth & Mimosaceae & 5 & & & & & $\mathrm{x}$ & $\mathrm{x}$ & $\mathrm{x}$ & $\mathrm{x}$ & $\mathrm{x}$ & \\
\hline Pentaclethra macroloba (Willd.) Kuntze & Mimosaceae & 1 & & & & & & & & & & $\mathrm{x}$ \\
\hline Pera glabrata (Schott) Poepp. ex Baill. & Euphorbiaceae & 4 & & & & & $\mathrm{x}$ & & $\mathrm{x}$ & $\mathrm{x}$ & $\mathrm{x}$ & \\
\hline Philodendron imbe Schott & Araceae & 1 & & & & & & & & & & $\mathrm{x}$ \\
\hline Pithecellobium amplissimum Ducke & Mimosaceae & 3 & & $\mathrm{x}$ & & $\mathrm{x}$ & & $\mathrm{x}$ & & & & \\
\hline Pithecellobium sp. 3 & Mimosaceae & 3 & & & & & & & & $\mathrm{x}$ & $\mathrm{x}$ & $\mathrm{x}$ \\
\hline Pithecellobium sp. 4 & Mimosaceae & 1 & & & & & & & & & & $\mathrm{x}$ \\
\hline
\end{tabular}

R. Árvore, Viçosa-MG, v.29, n.3, p.445-453, 2005 
Quadro 2 - cont.

Table 2 - cont.

\begin{tabular}{|c|c|c|c|c|c|c|c|c|c|c|c|c|}
\hline Espécie & Família & Freq & 1 & 2 & 3 & 4 & 5 & 6 & 7 & 8 & 9 & 10 \\
\hline Poecilanthe amazonica (Ducke) Ducke & Fabaceae & 1 & & & & & & & & & $\mathrm{x}$ & \\
\hline Polygonaceae Indet. & Polygonaceae & 3 & & & & $\mathrm{x}$ & & $\mathrm{x}$ & $\mathrm{x}$ & & & \\
\hline Psidium sp. 1 & Myrtaceae & 2 & & & & & & & & & $\mathrm{x}$ & $\mathrm{x}$ \\
\hline Psychotria lupulina Benth. & Rubiaceae & 8 & & & & $\mathrm{x}$ & $\mathrm{x}$ & $\mathrm{x}$ & $\mathrm{x}$ & $\mathrm{x}$ & $\mathrm{x}$ & $\mathrm{x}$ \\
\hline Psychotria sp. 1 & Rubiaceae & 1 & & & & & & & $\mathrm{x}$ & & & \\
\hline Quiina rhytidopus Tul. & Quiinaceae & 4 & & $\mathrm{x}$ & & $\mathrm{x}$ & $\mathrm{x}$ & & & $\mathrm{x}$ & & \\
\hline Salacia gigantea Loes. & Hippocrateaceae & 2 & & & & $\mathrm{x}$ & & & $\mathrm{x}$ & & & \\
\hline Salacia sp. 1 & Hippocrateaceae & 2 & & & & & & $\mathrm{x}$ & & & & $\mathrm{x}$ \\
\hline Scleria platensis & Cyperaceae & 8 & & $\mathrm{x}$ & $\mathrm{x}$ & & $\mathrm{x}$ & $\mathrm{x}$ & $\mathrm{x}$ & $\mathrm{x}$ & $\mathrm{x}$ & \\
\hline Solanum sp. 1 & Solanaceae & 1 & & & & & & & $\mathrm{x}$ & & & \\
\hline Solanum sp. 2 & Solanaceae & 1 & & & & & & & $\mathrm{x}$ & & & \\
\hline Swartzia argentea Spruce ex Benth & Fabaceae & 2 & & & & $\mathrm{x}$ & & & & & $\mathrm{x}$ & \\
\hline Swartzia laevicarpa Amshoff & Fabaceae & 1 & & & & & & & & & $\mathrm{x}$ & \\
\hline Swartzia polyphylla DC. & Fabaceae & 4 & & & & & $\mathrm{x}$ & $\mathrm{x}$ & $\mathrm{x}$ & $\mathrm{x}$ & & \\
\hline Tabebuia barbata (E. Mey) Sand. & Bignoniaceae & 3 & & & & $\mathrm{x}$ & $\mathrm{x}$ & & & & $\mathrm{x}$ & \\
\hline Symmeria paniculata Benth. & Polygonaceae & 3 & $\mathrm{x}$ & $\mathrm{x}$ & $\mathrm{x}$ & & & & & & & \\
\hline Talisia elephantipes Sand. ex Tutin & Sapindaceae & 1 & & & & & & & & & $\mathrm{x}$ & \\
\hline Tococa sp. 1 & Melastomataceae & 2 & & & & & & & $\mathrm{x}$ & & $\mathrm{x}$ & \\
\hline Tovomita macrophylla (Poepp.) Walp. & Clusiaceae & 7 & & & & $\mathrm{x}$ & $\mathrm{x}$ & $\mathrm{x}$ & $\mathrm{x}$ & $\mathrm{x}$ & $\mathrm{x}$ & $\mathrm{x}$ \\
\hline Tovomita sp. 1 & Clusiaceae & 1 & & & & & & & & & $\mathrm{x}$ & \\
\hline Trichilia sp. 1 & Meliaceae & 2 & & & & & $\mathrm{x}$ & $\mathrm{x}$ & & & & \\
\hline Trymatococcus sp. 1 & Moraceae & 1 & & & & & & & & & & $\mathrm{x}$ \\
\hline Unonopsis guatterioides (A.DC.) R.E. Fr. & Annonaceae & 1 & & & & & & & & & & $\mathrm{x}$ \\
\hline Virola elongata (Bent.) Warb. & Myristicaceae & 7 & & & & $\mathrm{x}$ & $\mathrm{x}$ & $\mathrm{x}$ & $\mathrm{x}$ & $\mathrm{x}$ & $\mathrm{x}$ & $\mathrm{x}$ \\
\hline Número total de espécies & & 9 & 12 & 11 & 16 & 19 & 25 & 34 & 28 & 33 & 25 & \\
\hline
\end{tabular}

Ferreira (2000) encontrou o mesmo conjunto de espécies em parcelas em cotas topográficas baixas em uma floresta de igapó não perturbada no rio Jaú, Amazonas, Brasil.

As clareiras situadas em posições mais altas no gradiente topográfica e sujeitas a períodos de inundação menores são, no entanto, principalmente colonizadas por espécies restritas às cotas topográficas mais altas da floresta de igapó, como Aldina latifolia, Caraipa grandifolia, Caryocar microcarpum, Swartzia polyphylla e Pentaclethra macroloba (Tabela 2), sendo a mesma composição florística encontrada em parcelas situadas em cotas topográficas médias e altas em uma floresta de igapó não perturbada no rio Jaú, Amazonas, Brasil (FERREIRA, 2000).

Concluindo, a riqueza e a composição específica da regeneração natural nas clareiras da floresta de igapó parecem estar diretamente relacionadas com a posição que têm no gradiente topográfico da área em estudo, resultado da capacidade diferencial que estas espécies têm para suportar o tempo de inundação.

\section{AGRADECIMENTOS}

Ao Departamento de Botânica do Instituto Nacional de Pesquisas da Amazônia (INPA), pela liberação do técnico em Botânica Sr. José Ferreira Ramos; à Nilzilene Cristo do Vale, do Museu Paraense Emílio Goeldi (MPEG), pela ajuda na digitação e formatação dos textos e tabelas deste estudo; e aos três revisores, por todas as críticas e sugestões. Este trabalho foi realizado com o suporte financeiro do Projeto INPA/MAX-Planck e da Fundação Botânica Margaret Mee.

\section{REFERÊNCIAS BIBLIOGRÁFICAS}

ADIS, J. Seasonal igapo-forests of Central Amazonian black-water rivers and their terrestrial arthropod fauna. In: SIOLI, H. (Ed.) The Amazon. Limnology and landscape ecology of a mighty tropical river and its basin. The Hague: 1984. v.6, p.246-268.

ADIS, J.; Furgh, K.; Irmler, U. Litter production of a central-amazonian black water inundation forest. Tropical Ecology, n.20, v.2, p.237-245. 1979. 
ALMEIDA, S. Clareiras naturais na Amazônia Central: Abundância, distribuição, estrutura e aspectos da colonização. 1989. 103f. Dissertação (Mestrado em Ecologia) Instituto Nacional de Pesquisas da Amazônia, Manaus, 1989.

AYRES, J. M. C. As matas de várzea do Mamirauá. MCT-CNPq-Programa do trópico úmido. Estudos do Mamirauá. 1993. 123f.

Sociedade civil de Mamirauá, Brasil.

AYRES, J.M.C. White Uakaris and

flooded forests. 1986. 338f. Tese (PhD em Ecologia). Cambridge University, Cambridge, 1986.

BROKAW, N. V. L. Treefalls, frequency, timming and consequences. In: LEIGH, E. G.; RAND, A. S.; WINDSON, D. M. (Eds.) The Ecology of a tropical forest: seasonal rhythms and long-term charges. Washington: Smith Institute Press, 1980.

BROKAW, N. V. L. The definiton of treefall gap and its effect on measure of forest dynamics. Biotropica, v.14, n.2, p.158-160, 1982.

BROKAW, N. V. L. Gap-phase regeration in a tropical forest. Ecology, v.66, n.3, p.682-687, 1985a.

BROKAW, N. V. L. Treefalls, regrowth, and community structure in tropical forest. In: PICKETT, S. T. A.; WHITE, P. S. (Eds). The Ecology of natural disturbance and patch dynamics. New York: Academic Press, 1985b.

CAMPBELL, D.G.; STONE, J. L.; JUNIOR, A. R. A comparison of the phytosociology and dynamics of three (várzea) forests of known ages, Rio Juruá, Western Brazilian Amazon. Journal of Linn. Society, v.108, p.213-237, 1992.

CARVALHO, L.M.T.; OLIVEIRA, A.T. Distribution, size and dynamics of canopy gaps in a cloud forest of the Ibitipora Range, south-eastern Brazil. In: GOTTSBERGER, G.; LIEDE, S. (Eds) Life forms of tropical forests. 2001. 346.f. Dissertationes (M.S. Botanicae) Cramer in der Gebruder Borntraeger Verlagsbuchhandlung, BerlinStuttgart, 2001.

R. Árvore, Viçosa-MG, v.29, n.3, p.445-453, 2005
DUCKE, A.; Black, G. A. Notas sobre a fitogeografia da Amazônia brasileira. Boletim Técnico Instituto Agronômico Norte, v.29, p.1-69. 1953.

FERREIRA, L.V. Effect of flooding duration on species richness, floristic composition and forest structure in river margin habitats in Amazonian blackwater floodplain forests: Implications for future Design of protected areas. Biodiversity and Conservation, v.9, p.1-14, 2000.

FERREIRA, L.V.; STOHLGREN, T.J. Effects of river level fluctuation on plant species richness, diversity, and distribution in a floodplain forest in central Amazonia. Oecologia, v.120, n.4, p.582-587.

FERREIRA, L.V. Floristic composition and structure of the three hectares in a floodplain forest in the Brazilian Central Amazon.

Biodiversity and Conservation, v.6, n.10, p.1355-1365, 1997.

FERREIRA, L.V. O efeito do período de inundação na zonação de comunidades, fenologia e regeneração em uma floresta de igapó na Amazônia Central. 1991. 161f. Dissertação (Mestrado em Ecologia). Instituto Nacional de Pesquisas da Amazônia, Manaus, 1991.

FITTKAU E.J. Ökologische Gliederung des Amazonas-Gebietes auf Geochemischer Grundlage. Münster, Forsch. Geologischies Palãontol, v.20/21, p.35-50, 1971.

HARTSHORN, G. S. Treefalls and tropical forest dynames. In: TOMLINDON, P. B.; ZIMMERMANN, M. N. (Eds). Tropical trees as living systems. Cambridge: Cambridge University Press, 1978. p.617-638.

JUNK, W. J. Flood tolerance and tree distribution in central Amazonia. In: HOLM-NIELSEN L. B.; NIELSEN I. C.; BALSLEV. H. (Eds.) Tropical Forest Botanical Dynamics. Speciation and Diversity. London: Academic Press, 1989. p.47-64.

KEEL, S.H.; PRANCE, G.T. Studies of the vegetation of a black water igapó (rio Negro Brazil). Acta Amazônica, v.9, p.645-655, 1979. 
MARTINS, S.V; RODRIGUES, R.R. Produção de serapilheira em clareiras de uma floresta estacional semidecidual no município de Campinas, SP. Revista Brasileira de Botânica, v.22, n.3, p.1-13, 1999.

OLIVEIRA-FILHO, F.J.B. Padrão de desmatamento e evolução da estrutura da paisagem em Alta Floresta (MT). 2001. 127f. Dissertação (Mestrado em Ecologia). Universidade de São Paulo, São Paulo, 2001.

PIEDADE, M.T.F. Ecologia e biologia reprodutiva de Astrocaryum jauari Mart. (Palmae) como exemplo de população adaptada às áreas inundáveis do rio Negro (igapós).1985. 187f. Dissertação (Mestrado em Ecologia). Instituto Nacional de Pesquisas da Amazônia, Manaus, 1985.

PIRES, J.M.; Prance, G.T. The vegetation types of the Brazilian Amazon. In: G.T. PRANCE AND T.E. LOVEJOY (Eds.). Amazonia: Key Environment. London: Pergamon Press, 1985. p.109-145.

PRANCE, G.T. Notes on vegetation of Amazonia III. The terminology of Amazonian forest types subject to inundation. Brittonia, v.31, p.26-38, 1979.
RODRIGUES, W. A. Estudo preliminar na mata de várzea alta de uma ilha do baixo rio Negro de solo argiloso e úmido. Publicação Avulsa do INPA - Série Botânica, v.10, p.50, 1961.

SALO J., K. R. et al. River dynamics and the diversity of Amazon lowland forest. Nature, v.322, p.254-258, 1986.

TABARELLI, M.; MANTOVANI, W. A regeneração de uma floresta tropical montana após corte e queima (São Paulo - Brasil).

Revista Brasileira de Biologia, v. 59, n.2, p. 239-251, 1999.

TAKEUCHI, M. The structure of the Amazonian vegetation. VI. Igapó. Journal Faculty Scicence of University of Tokyo, v.8, n.7, p.297-304, 1962.

UHL, C. K.; DEZZEO, N.; MAQUIRINO, P. Vegetation dynamics in Amazonian treefall gaps. Ecology, v.69, p.751-763, 1988.

WHITMORE, T. C. Gaps in the forest canopy. In: TOMLINSON, P. B.; ZIMMERMANN, M. H. (Eds.) Tropical trees as living systems. New York: Cambridge University Press, 1978, p. 639-655.

WHITMORE, T. C. Tropical rain forest of the Far East. Oxford: Oxford University Press, p.352, 1985. 